\title{
MÓDULO DE ELASTICIDAD COMO CRITERIO DE REGULACIÓN HÍDRICA DEL FREJOL COMÚN (PHASEOLUS VULGARIS L.) BAJO REDUCCIONES CONTROLADAS DE RIEGO
}

\section{Cristhian Vega Ponce y Jesús Abel Mejía Marcacuzco}

\section{RESUMEN}

El propósito de esta investigación consistió en estudiar el efecto de reducciones controladas de riego sobre el módulo de elasticidad $\left(\varepsilon_{\mathrm{v}}\right)$ en plantas de frejol común (Phaseolus vulgaris L.) cultivadas en maceteros de respuesta hidrogravitrópica. Las plantas fueron sometidas a riego completo de raíces (RCR) y riego parcial de raíces (RPR), donde el agua asignada de acuerdo a la curva de retención agua-suelo permitió controlar y configurar cuatro tratamientos (RPR300, RPR500, RCR300 y RCR500 o control). Se monitoreó el potencial hídrico xilemático (Чx) de las hojas, para luego construir la curva presión-volumen (P-V) y determinar $\varepsilon_{\mathrm{v}}$. Los resultados mostraron que los diferentes volúmenes de agua aplicados generaron importantes variaciones en los niveles de $\varepsilon_{v}$; sin embargo, en los tratamientos configurados para llevar el suelo a capacidad de campo (RPR500 y RCR500) fue donde se obtuvieron los mejores desempeños de $\varepsilon_{\mathrm{v}}$, efecto esperado principalmente antes de aplicar el riego a las plantas $(15,63$ y 15,34 MPA, respectivamente). Finalmente, aunque ambos tratamientos obtuvieron el mismo nivel de significancia de $\varepsilon_{v}$, RPR500 se destacó sobre el tratamiento control, porque los volúmenes de agua reducidos, combinados con el mantenimiento de diferentes valores de humedad en el suelo explorado por las raíces, pudieron ser claves en el favorecimiento de un ajuste elástico.

Palabras claves: Estrés Hídrico, Módulo de Elasticidad, Riego Parcial de Raíces.

DOI: 10.23881/idupbo.017.1-2i 